\title{
Quality Process Measures for Rheumatoid Arthritis: Performance from Members Enrolled in a National Health Plan
}

\author{
Joseph Tkacz, MS; Lorie A. Ellis, PhD; Roxanne Meyer, PharmD; Susan C. Bolge, PhD; \\ Brenna L. Brady, PhD; and Charles Ruetsch, PhD
}

\begin{abstract}
BACKGROUND: Health care quality problems are reflected in the underuse, overuse, and misuse of health care services. There is evidence suggesting that the quality of rheumatoid arthritis (RA) patient care is suboptimal, which has spurred the development of a number of systematic quality improvement metrics.
\end{abstract}

OBJECTIVE: To investigate a quality process measurement set in a sample of commercially insured RA patients.

METHODS: Medical, pharmacy, and laboratory claims for members with an RA diagnosis (ICD-9-CM 714.x) during calendar years 2008 through 2012 were extracted from the Optum Clinformatics Data Mart database. Eight process quality measures focused on RA patient response and tolerance to therapy were examined in the claims database. Measures were calculated for individual calendar years from 2009 to 2012, inclusive.

RESULTS: The majority of adult RA patients received at least 1 prescription for a disease-modifying antirheumatic drug (DMARD) across the 4 measurement years: range $=78.5 \%-81.6 \%$. Erythrocyte sedimentation rate and C-reactive protein testing were also evident in the majority of the sample, with $67.1 \%-72.2 \%$ of newly diagnosed RA patients receiving baseline testing, and $56.0 \%-58.7 \%$ of existing RA patients receiving annual testing. Among methotrexate users, liver function tests were performed in $74.5 \%-75.7 \%$ of treated patients, serum creatinine tests in $70.1 \%-72.6 \%$ of patients, and complete blood count tests in $74.5 \%-76.0 \%$ of patients. Additionally, most patients initiating a new DMARD had a claim for a baseline serum creatinine test $(68.0 \%-70.3 \%)$ and baseline liver function test (69.3\%-71.0\%).

CONCLUSIONS: Findings suggest that a majority of RA patients are attaining patient quality process measures, although a considerable proportion of patients (approximately 25\%) may be receiving suboptimal care. Further studies are warranted to understand whether attainment of these measures translates into better outcomes.

J Manag Care Pharm. 2015;21(2):135-43

Copyright $\odot 2015$, Academy of Managed Care Pharmacy. All rights reserved.

\section{What is already known about this subject}

In the United States, the annual societal costs of rheumatoid arthritis (RA) have been estimated at $\$ 128$ billion.

Although the treatment of RA has improved significantly with the development of a variety of biologic agents, some evidence suggests the quality of RA patient care remains suboptimal.

Efforts to improve quality in health care have utilized a number of approaches, including the redesign of continuing professional education, improved physician practice peer review, and the systematic measurement of quality improvement.

\section{What this study adds}

This study investigated the rate of RA quality process measurement achievement in a national sample of commercially insured patients diagnosed with RA.

Overall, a majority of the sample eligible for inclusion in the measure calculations met the requirements for these indicators, with attainment rates ranging from $56.0 \%-81.6 \%$ across 4 years.

Although results are encouraging, nearly $25 \%$ of patients may be receiving suboptimal care, suggesting potential need for increased patient or physician awareness.

$\mathrm{R}$ heumatoid arthritis (RA) is an autoimmune disease characterized by chronic inflammation of the synovial joints and surrounding tissues; in severe cases, RA can progress and involve other organ systems. ${ }^{1}$ It is estimated that nearly 50 million U.S. adults suffer from some form of arthritis, ${ }^{2}$ with 1.3 million suffering specifically from RA. ${ }^{3}$ In the United States alone, the annual societal costs of RA have been estimated at $\$ 128$ billion. $^{4}$

Health care quality problems impact patients with chronic illnesses as reflected in the underuse, overuse, and misuse of services. ${ }^{5}$ The Institute of Medicine has famously defined quality in health care as "the degree to which health services for individuals and populations increase the likelihood of desired health outcomes and are consistent with current professional knowledge." Although the treatment of RA has improved significantly over the past decade with the development of a variety of biologic agents targeting molecules critical to chronic inflammation (e.g., tumor necrosis factor $\mathrm{alpha}^{7}$ ), some evidence suggests that the quality of RA patient care remains suboptimal, ${ }^{8-10}$ particularly among the geriatric population. ${ }^{11,12}$ For this reason, a number of quality improvement measures have been developed to assess the various components of RA treatment.

Efforts to improve quality in health care over the past 50 years have utilized a number of approaches, including the redesign of continuing professional education, improved physician practice peer review, and the systematic measurement of quality improvement. ${ }^{13}$ The American College of Rheumatology, ${ }^{14}$ the American Medical Association, ${ }^{15}$ and the National Quality Forum $(\mathrm{NQF})^{16}$ have developed their own recommendations 
for quality measures pertaining to RA. To date, a comprehensive examination of the feasibility and overall adoption rate of the NQF RA indicators has yet to be undertaken. Using administrative claims data, this exploratory study investigated process quality measure achievement in a national sample of commercially insured patients diagnosed with RA. The specific metrics investigated were adopted by the NQF in December 2009 and were endorsed through July 2014.

\section{Methods}

\section{Study Sample}

Study data derived from the Optum Clinformatics Data Mart database (OptumInsight Life Sciences, Inc., Eden Prairie, MN). The Clinformatics Data Mart is a database of administrative health claims for members of a large national managed care company. Medical, pharmacy, and laboratory claims for members with a diagnosis of RA (International Classification of Diseases, Ninth Revision, Clinical Modification [ICD-9-CM] 714.x) during calendar years 2008 through 2012 were extracted to assess quality measurement feasibility and achievement. All data were void of identifying information in compliance with the Health Insurance Portability and Accountability Act of 1996. Therefore, institutional review board approval was unnecessary for this study.

\section{Measures}

Table 1 contains the official descriptions of the 8 RA quality process measures examined in this study, which were selected based on calculation feasibility within a claims database. ${ }^{16}$ Each measure is calculated over an individual calendar year. The calculation of these measures using administrative claims data requires the computation of the following 2 components for each indicator:

1. Denominator statement: The denominator consists of the number of patients aged more than 18 years meeting the criteria for an RA diagnosis during either the measurement year, or in the case of measures examining existing RA patients, in the previous year(s). Denominator statements will also specify an additional inclusion criterion based on the particular type of patient the measure was designed to assess (e.g., new disease-modifying antirheumatic drug [DMARD] initiator or existing methotrexate user).

2. Numerator statement: The numerator for each measure will be a subset of patients in the denominator who receive some form of treatment or lab test that is indicative of quality patient care.

The claims-based logic used to calculate each measure is described in the following paragraphs. For all denominator statements, patients were required to be continuously eligible for the entire year and must have met the following criteria for a diagnosis of RA during a given year: Two claims on 2 separate dates with (a) a primary diagnoses of RA (ICD-9-CM 714.x) and (b) a place of service code for either "office" or "outpatient hospital."

For measures requiring the detection of ambulatory or oral DMARD utilization, the "brand name" field in the pharmacy table was used to identify relevant medications. For measures 0589 and 0590, which did not restrict DMARD usage to ambulatory fills, relevant J-codes appearing in the "procedure code" field on the medical record were used in addition to the pharmacy record. For measures requiring the detection of laboratory tests, appropriate Current Procedural Terminology (CPT) codes appearing on the medical record were used. The laboratory results table was not used in any of the calculations, since the measures were designed to consider billing claims only. Finally, all measures also included the exclusionary criteria defined by the NQF (for a complete description of measure definitions, visit www.qualityforum.org). The list of the drug brand names, generic names, J-codes, and CPT codes used to calculate these measures may be viewed in the Appendix, available in the online article. All measures were calculated over calendar years 2009-2012.

Measure 0054: DMARD Therapy. This measure was designed to assess the frequency in which ambulatory DMARD prescriptions were filled. Members were included in the denominator statement if they met the RA diagnosis criterion and were continuously eligible for the entire measurement year. Members were included in the numerator if they filled 1 ambulatory prescription for any DMARD between January 1 and November 30 of the measurement year.

Measure 0589: New DMARD Baseline Serum Creatinine Testing. This measure was designed to assess the frequency in which appropriate baseline serum creatinine testing was performed after a new start of select DMARDs. Members in the denominator must have been continuously eligible for 2 yearsthe measurement year and the year prior to the measurement year-and must have also met the RA diagnosis criterion in any of the 3 most recent available calendar years starting with the measurement year. Members must also have been newly treated with either methotrexate, leflunomide, azathioprine, D-Penicillamine, intramuscular gold, cyclosporine, or cyclophosphamide during the measurement year. A new treatment start was operationally defined as the first appearance of any of the aforementioned DMARDs preceded by a 1-year treatmentnaive period. Members were included in the numerator if baseline serum creatinine testing was performed within 90 days before or 14 days after the new DMARD initiation.

Measure 0590: New DMARD Baseline Liver Function Test. This measure was designed to assess the frequency in which appropriate baseline liver function testing was performed after a new start of select DMARDs. Members in the denominator must have been continuously eligible for 2 years-the measurement year and the year prior to the measurement year- 


\section{TABLE 1 Quality Process Rheumatoid Arthritis Measures}

\begin{tabular}{l|l|}
\hline Measure & \multicolumn{1}{|c|}{ Measure Description } \\
\hline 0054 DMARD & $\begin{array}{l}\text { Percentage of patients aged 18 years and } \\
\text { older diagnosed with RA who have had at } \\
\text { therapy in RA } \\
\text { least 1 ambulatory prescription dispensed } \\
\text { for a DMARD. }\end{array}$
\end{tabular}

Numerator Statement
prescription dispensed for a DMARD during the measurement year.

Denominator Statement
All patients, aged $\geq 18$ years as of
December 31 of the measurement year,
with a diagnosis RA. Two face-to-face
physician encounters with an RA diag-
nosis with different dates of service in an
ambulatory or nonacute inpatient setting
between January 1 and November 30 of
the measurement year are required to con-
firm an RA diagnosis.
Patients aged

\section{RA: new} DMARD baseline serum creatinine test

0590 RA: new DMARD baseline LFT
This measure identifies adult patients with a diagnosis of RA who received appropriate baseline serum creatinine testing within 90 days before to 14 days after the new start of methotrexate, leflunomide, azathioprine, D-Penicillamine, intramuscular gold, cyclosporine, or cyclophosphamide during the measurement year.

This measure identifies adult patients with a diagnosis of RA who received appropriate baseline LFT (AST or ALT) within 90 days before to 14 days after the new start of sulfasalazine, methotrexate, leflunomide, azathioprine, cyclosporine, or cyclophosphamide during the measurement year.

0592 RA: annual ESR or CRP

This measure identifies adult patients with a history of RA who have received ESR or CRP lab tests during the measurement year.

0601 New RA baseline ESR or CRP within 3 months

\section{This measure identifies adult patients} newly diagnosed with RA during the first 8 months of the measurement year who received ESR or CRP lab tests either 4 months (3 months + 1-month grace period) before or after the initial diagnosis.

0597 Methotrexate: $\quad$ This measure identifies adult patients LFT within 3 months with RA who were prescribed at least a 6-month supply of methotrexate during the measurement year and received an LFT in the 120 days ( 3 months + 1-month grace period) following the earliest observed methotrexate prescription claim.

0598 Methotrexate: CBC within 3 months

This measure identifies adult patients with RA who were prescribed at least a 6-month supply of methotrexate during the measurement year and received a CBC test within 120 days (3 months +1 -month grace period) following the earliest observed methotrexate prescription claim.

0599 Methotrexate: creatinine within 3 months
This measure identifies adult patients with RA who were prescribed at least a 6-month supply of methotrexate during the measurement year and received a serum creatinine test in the 120 days ( 3 months + 1-month grace period) after the earliest observed methotrexate prescription claim.
Patients in the denominator who received serum creatinine testing within 90 days before to 14 days after the new start of methotrexate, leflunomide, azathioprine, D-Penicillamine, intramuscular gold, cyclosporine, or cyclophosphamide during the measurement year.

Patients in the denominator who received liver function testing within 90 days before to 14 days after the new start of sulfasalazine, methotrexate, leflunomide, azathioprine, cyclosporine, or cyclophosphamide during the measurement year.

Patients in the denominator who had ESR or CRP lab tests during the measurement year.

Patients in the denominator who had ESR or CRP lab tests either 4 months before or after the initial RA diagnosis date.

Patients in the denominator who received an LFT within 120 days following the earliest observed methotrexate prescription claim.
Patients aged $\geq 18$ years with a history of RA and a new start of methotrexate, leflunomide, azathioprine, D-Penicillamine, intramuscular gold, cyclosporine, or cyclophosphamide any time from the beginning of the measurement year to 14 days prior to the end of the measurement year.

Patients aged $\geq 18$ years with a history of RA and a new start of sulfasalazine, methotrexate, leflunomide, azathioprine, cyclosporine, or cyclophosphamide any time from the beginning of the measurement year to 14 days prior to the end of the measurement year.

Patients aged $\geq 18$ years with a history of RA diagnosed prior to the measurement year.

Patients aged $\geq 18$ years newly diagnosed with RA during the first 8 months of the measurement year.
Patients in the denominator who received a CBC test within 120 days following the earliest observed methotrexate prescription claim.
Patients aged $\geq 18$ years with RA who have received at least a 6 -month supply of oral methotrexate during the measurement year.
Patients aged $\geq 18$ years with RA who have received at least a 6-month supply of oral methotrexate during the measurement year.
Patients in the denominator who received a serum creatinine or BUN test in the 120 days following the earliest observed methotrexate prescription claim.
Patients aged $\geq 18$ years with RA who have received at least a 6-month supply of oral methotrexate during the measurement year.

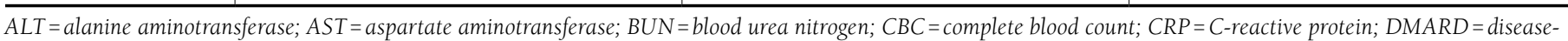
modifying antirheumatic drug; ESR=erythrocyte sedimentation rate; LFT=liver function test; $R A=$ rheumatoid arthritis. 
and must have also met the RA diagnosis criterion in any of the 3 most recent available calendar years starting with the measurement year. Members must also have been newly treated with either sulfasalazine, methotrexate, leflunomide, azathioprine, cyclosporine, or cyclophosphamide during the measurement year. A new treatment start was operationally defined as the first appearance of any of the aforementioned DMARDs preceded by a 1-year treatment-naive period. Members were included in the numerator if baseline liver function testing was performed within 90 days before or 14 days after the new DMARD initiation.

Measure 0592: Annual Erythrocyte Sedimentation Rate or C-Reactive Protein. This measure was designed to assess the frequency in which patients with a history of RA receive annual erythrocyte sedimentation rate (ESR) or C-reactive protein (CRP) tests. Members in the denominator must have been continuously eligible for the entire measurement year and must have also met the RA diagnosis criterion in any of the 3 most recent available calendar years preceding the measurement year. Members were included in the numerator if they received ESR or CRP tests any time during the measurement year.

Measure 0601: New RA Baseline ESR or CRP Within 3 Months. This measure was designed to assess the frequency in which new RA patients receive appropriate ESR or CRP tests. Members in the denominator must have been continuously eligible for the entire measurement year and the year prior to the measurement year. Additionally, members must have also met the RA diagnosis criterion during the first 8 months of the calendar year and may not have met the RA diagnosis criterion in the 2 most recently available calendar years prior to the measurement year. Members were included in the numerator if they received ESR or CRP tests within 120 days of the earliest RA diagnosis appearing in the measurement year.

Measure 0597: Methotrexate-Liver Function Test Within 3 Months. This measure was designed to assess the frequency in which RA patients treated with methotrexate receive liver function tests. Members in the denominator must have been continuously eligible for the entire measurement year and must have met the RA diagnosis criterion during the measurement year. Additionally, members must have received a minimum of a 6-month supply of oral methotrexate during the measurement year. Members were included in the numerator if they received a liver function test within 120 days following the earliest observed methotrexate prescription claim.

Measure 0598: Methotrexate-Complete Blood Count Test Within 3 Months. This measure was designed to assess the frequency in which RA patients treated with methotrexate receive a complete blood count $(\mathrm{CBC})$ test. Members in the denominator must have been continuously eligible for the entire measurement year and must have met the RA diagnosis criterion during the measurement year. Additionally, members must have received a minimum of a 6-month supply of oral methotrexate during the measurement year. Members were included in the numerator if they received a complete blood $\mathrm{CBC}$ test within 120 days following the earliest observed methotrexate prescription claim.

Measure 0599: Methotrexate-Creatinine Within 3 Months. This measure was designed to assess the frequency in which RA patients treated with methotrexate receive serum creatinine or blood urea nitrogen (BUN) tests. Members in the denominator must have been continuously eligible for the entire measurement year and must have met the RA diagnosis criterion during the measurement year. Additionally, members must have received a minimum of a 6-month supply of oral methotrexate during the measurement year. Members were included in the numerator if they received a serum creatinine or BUN test within 120 days following the earliest observed methotrexate prescription claim.

For measures examining history of RA $(0589,0590,0592)$ or absence of RA to identify new patients (0601), a minimum of 2 years of eligibility was imposed on the sample (the measurement year and the year prior to the measurement year), since these 2 years were essential to correctly calculating measures. However, an RA diagnosis look-back period of a maximum of 3 years was utilized for those patients with available claims dating back further. This method allowed for the identification of those RA patients who may have been out of treatment for an extended period of time, which may have incorrectly resulted in their inclusion/exclusion in these measures. This method was employed as an extra level of assurance that as many "correct new" or "correct existing" RA patients as possible were captured for the respective measures-again, given that some may have been out of treatment for some time.

\section{Demographic Characteristics}

For each calendar year in which the quality measures were reported (2009-2012), patient demographics were summarized from the membership table. Measures included age, gender, geographic region of residence, insurance line of business, and type of benefit plan. Additionally, the Charlson Comorbidity Index, an overall measure of health, was calculated during each measurement year, as were the rates for a variety of other comorbidities of interest. ${ }^{17}$ The Charlson Comorbidity Index was developed to predict mortality and is calculated by summing the number of comorbid diagnoses (weighted by severity) appearing during a specified period of time. A greater number of comorbidities results in a higher score and is an indication of lower overall health. ${ }^{17}$ Given that each quality measure contains specific inclusion and exclusion criteria resulting in the creation of a unique sample for each measure for each year, analyses of demographics included all members meeting the age and RA diagnosis inclusion criteria for a given year. 


\begin{tabular}{|c|c|c|c|c|c|c|c|c|}
\hline \multirow[b]{2}{*}{ Demographic Characteristics } & \multicolumn{2}{|c|}{$\mathrm{CY} 2009, \mathrm{~N}=32,956$} & \multicolumn{2}{|c|}{$\mathrm{CY} 2010, \mathrm{~N}=31,795$} & \multicolumn{2}{|c|}{ CY2011, $N=32,044$} & \multicolumn{2}{|c|}{$\mathrm{CY} 2012, \mathrm{~N}=31,419$} \\
\hline & $\mathrm{M} / f$ & $\mathrm{SD} / \%$ & $\mathrm{M} / f$ & $\mathrm{SD} / \%$ & $\mathrm{M} / f$ & $\mathrm{SD} / \%$ & $\mathrm{M} / f$ & $\mathrm{SD} / \%$ \\
\hline Female & 25,045 & 76.0 & 23,999 & 75.5 & 24,142 & 75.3 & 23,695 & 75.4 \\
\hline Age & 53.1 & 12.4 & 52.8 & 12.0 & 52.8 & 12.0 & 52.8 & 12.0 \\
\hline \multicolumn{9}{|l|}{ Geographic region } \\
\hline Mid-American & 12,943 & 39.3 & 12,985 & 40.8 & 13,550 & 42.3 & 13,660 & 43.5 \\
\hline Northeast & 2,737 & 8.3 & 2,764 & 8.7 & 2,677 & 8.4 & 2,597 & 8.3 \\
\hline Southeast & 11,869 & 36.0 & 10,719 & 33.7 & 10,742 & 33.5 & 10,077 & 32.1 \\
\hline West & 5,400 & 16.4 & 5,318 & 16.8 & 5,070 & 15.8 & 5,078 & 16.2 \\
\hline \multicolumn{9}{|l|}{ Benefit plan business type } \\
\hline Commercial & 32,061 & 97.3 & 31,154 & 98.0 & 32,033 & 99.9 & 31,413 & 99.9 \\
\hline Medicare/Medicaid & 895 & 2.7 & 641 & 2.0 & 11 & 0.1 & 6 & 0.1 \\
\hline Charlson Comorbidity Index & 0.72 & 1.27 & 0.68 & 1.20 & 0.67 & 1.18 & 0.66 & 1.16 \\
\hline \multicolumn{9}{|l|}{ Specific comorbidites } \\
\hline Chronic pulmonary disease & 5,361 & 16.3 & 5,012 & 15.8 & 4,885 & 15.2 & 4,805 & 15.3 \\
\hline Mild liver disease & 1,607 & 4.9 & 1,461 & 4.6 & 1,516 & 4.7 & 1,503 & 4.8 \\
\hline Diabetes without complications & 3,820 & 11.6 & 3,648 & 11.5 & 3,578 & 11.2 & 3,481 & 11.1 \\
\hline Depression & 3,668 & 11.1 & 3,516 & 11.1 & 3,618 & 11.3 & 3,585 & 11.4 \\
\hline Osteoarthritis & 19,133 & 58.1 & 18,221 & 57.3 & 18,570 & 58.0 & 18,297 & 58.2 \\
\hline Chronic pain & 1,794 & 5.4 & 1,963 & 6.2 & 2,293 & 7.2 & 2,468 & 7.9 \\
\hline Osteoporosis & 5,127 & 15.6 & 4,373 & 13.8 & 4,002 & 12.5 & 3,586 & 11.4 \\
\hline Hypothyroidism & 5,381 & 16.3 & 5,332 & 16.8 & 5,605 & 17.5 & 5,450 & 17.3 \\
\hline Disorders of lipid metabolism & 12,119 & 36.8 & 11,610 & 36.5 & 11,754 & 36.7 & 11,200 & 35.6 \\
\hline Hypertension & 13,228 & 40.1 & 12,496 & 39.3 & 12,539 & 39.1 & 12,043 & 38.3 \\
\hline
\end{tabular}

Note: Yearly samples are composed of patients aged $>18$ years meeting the definition of rheumatoid arthritis sometime during the measurement year. $C Y=$ calendar year; $M / f=$ mean/frequency; $S D=$ standard deviation.

\section{Data Analysis}

Descriptive statistics were reported for all demographic measures, including means and standard deviations for scale variables, frequencies, and proportions for categorical variables. For each process quality metric, numerator and denominator frequencies were reported, in addition to the proportions of patients achieving each quality measure per year. All data management and analyses were conducted using SPSS v.20 (SPSS Inc., Chicago, IL).

\section{Results}

Sample characteristics in each measurement year are shown in Table 2. All demographic characteristics assessed were consistent across the 4 measurement years. Approximately 75\% of the sample was female (range $=75.3 \%-76.0 \%$ ), with a mean age of early fifties (52.8-53.1 years). In addition, the majority of the members were residents of the southeastern and middle regions of the United States (74.5\%-75.8\%), and nearly all had commercial coverage (more than 97\%). Osteoarthritis, hypertension, and disorders of lipid metabolism were the most prevalent comorbid conditions observed in this sample.

Quality process measure achievement by year are presented in Table 3. The majority of adult RA patients received at least 1 prescription for a DMARD (measure 0054) across the 4 mea- surement years (range $=78.5 \%-81.6 \%)$. The majority of patients initiating a new DMARD received appropriate baseline serum creatinine testing, 68.0\%-70.3\% (measure 0589) and baseline liver function testing, 69.3\%-71.0\% (measure 0590). ESR and CRP testing were also evident in the majority of the sample. Between 56.0\%-58.7\% of existing RA patients received their annual ESR or CRP test (measure 0592), while 67.0\%-72.2\% of newly diagnosed RA patients received baseline ESR or CRP testing within 3 months of the initial diagnosis (measure 0601). Finally, utilization of appropriate lab tests among methotrexate users was also high, with between $74.5 \%-75.7 \%$ receiving liver function tests (measure 0597), 74.5\%-76.0\% receiving CBC tests (measure 0598), and 70.1\%-72.6\% receiving serum creatinine tests (measure 0599).

\section{Discussion}

RA is a systemic autoimmune disease characterized by excessive inflammation that primarily manifests in synovial joints but can occur in other tissues of the body. Treatment of RA is aimed at reducing inflammation leading to reduced pain, increasing quality of life, and preventing irreversible damage to joints and tissues. The purpose of this exploratory study was to examine RA quality process measures in a commercially insured, national sample of RA patients. These measures were 


\begin{tabular}{|c|c|c|c|c|}
\hline \multirow{2}{*}{$\begin{array}{l}\text { TABLE } 3 \\
\text { Measure }\end{array}$} & \multicolumn{4}{|c|}{$\begin{array}{l}\text { Measurement Achievement } \\
\text { by Calendar Year }\end{array}$} \\
\hline & 2009 & 2010 & 2011 & 2012 \\
\hline \multicolumn{5}{|c|}{ 0054: DMARD therapy for RA } \\
\hline Numerator & 21,072 & 20,222 & 21,157 & 21,108 \\
\hline Denominator & 26,832 & 25,244 & 26,184 & 25,882 \\
\hline Percentage & 78.5 & 80.1 & 80.8 & 81.6 \\
\hline \multicolumn{5}{|c|}{ 0589: New DMARD baseline serum creatinine } \\
\hline Numerator & 1,852 & 1,824 & 1,844 & 1,942 \\
\hline Denominator & 2,724 & 2,646 & 2,646 & 2,761 \\
\hline Percentage & 68.0 & 68.9 & 69.7 & 70.3 \\
\hline \multicolumn{5}{|c|}{ 0590: New DMARD baseline LFT } \\
\hline Numerator & 2,083 & 2,108 & 2,086 & 2,190 \\
\hline Denominator & 3,005 & 2,970 & 2,937 & 3,092 \\
\hline Percentage & 69.3 & 71.0 & 71.0 & 70.8 \\
\hline \multicolumn{5}{|c|}{ 0592: Annual ESR or CRP } \\
\hline Numerator & 11,137 & 12,076 & 13,291 & 13,638 \\
\hline Denominator & 18,974 & 21,164 & 23,743 & 24,198 \\
\hline Percentage & 58.7 & 57.1 & 56.0 & 56.4 \\
\hline \multicolumn{5}{|c|}{ 0601: New RA Baseline ESR or CRP within 3 Months } \\
\hline Numerator & 3,091 & 2,806 & 2,685 & 2,737 \\
\hline Denominator & 4,607 & 3,887 & 3,734 & 3,811 \\
\hline Percentage & 67.1 & 72.2 & 71.9 & 71.8 \\
\hline \multicolumn{5}{|c|}{ 0597: Methotrexate: LFT within 3 Months } \\
\hline Numerator & 6,206 & 6,093 & 6,092 & 6,356 \\
\hline Denominator & 8,315 & 8,099 & 8,177 & 8,396 \\
\hline Percentage & 74.6 & 75.2 & 74.5 & 75.7 \\
\hline \multicolumn{5}{|c|}{ 0598: Methotrexate: $\mathrm{CBC}$ within 3 Months } \\
\hline Numerator & 6,237 & 6,107 & 6,094 & 6,378 \\
\hline Denominator & 8,315 & 8,099 & 8,177 & 8,396 \\
\hline Percentage & 75.0 & 75.4 & 74.5 & 76.0 \\
\hline \multicolumn{5}{|c|}{ 0599: Methotrexate: Creatinine within 3 Months } \\
\hline Numerator & 5,813 & 5,733 & 5,775 & 6,087 \\
\hline Denominator & 8,296 & 8,086 & 8,164 & 8,385 \\
\hline Percentage & 70.1 & 70.9 & 70.7 & 72.6 \\
\hline \multicolumn{5}{|c|}{$\begin{array}{l}C B C=\text { complete blood count } ; C R P=C \text {-reactive protein } ; D M A R D=\text { disease-modify- } \\
\text { ing antirheumatic drug; } E S R=\text { erythrocyte sedimentation rate; } L F T=\text { liver function } \\
\text { test } ; R A=\text { rheumatoid arthritis. }\end{array}$} \\
\hline
\end{tabular}

endorsed by the NQR from 2009 to 2014 and were designed as tools for physicians to objectively measure and document patient response and tolerance to therapy over time. This is the first peer-reviewed examination of the relevance of these measures with an application to administrative claims data.

The overall attainment of the 8 quality measures examined in the present study was high, with rates ranging from $56.0 \%$ 81.6\%. Patterns emerged among those measures assessing similar aspects of treatment. Measures assessing components of DMARD therapy, specifically methotrexate use (measures 0054, 0597, 0598), were achieved by the highest proportion of patients (74.5\%-81.6\%). Measures assessing new treatment pathways, such as a new DMARD prescription or a new RA patient (measures 0589, 0590, 0601), were achieved by a slightly lower rate (67.1\%-72.2\%), albeit still the majority. The measure assessing annual ESR or CRP testing of existing RA patients (measure 0592) was achieved by the lowest proportion of patients (56.0\%-58.7\%).

DMARDs are considered the standard of care for RA treatment; therefore, the proportion of patients filling at least 1 ambulatory prescription for a DMARD (measure 0054) was expected to be achieved at the highest rate (78.5\%-81.6\%). ${ }^{18}$ It is worth noting that this measure does not currently include patients receiving intravenously administered biologic DMARDs, such as infliximab, abatacept, and tocillizumab, so the true number of DMARD-treated patients in this sample is likely somewhat greater than reported. Indicators assessing $\mathrm{CBC}$ and liver function testing with methotrexate (among 1 of the most commonly prescribed DMARDs) were also met at a high rate $(74.5 \%-75.7 \%$ and $74.5 \%-76.0 \%$, respectively; measures 0597 and 0598). Creatinine testing within 12 weeks of initiation of methotrexate (measure 0599) was achieved by fewer patients (70.1\%-72.6\%). Although each of these measures were attained by the majority of eligible methotrexate users, a gap remains between the completion of $\mathrm{CBC}$ and liver function testing compared with creatinine testing. The most common side effects of methotrexate involve the hematologic and hepatic systems. ${ }^{19,20}$ The reduced percentage of patients receiving creatinine testing compared with liver function testing is consistent with the relative rates of organ system involvement-approximately 30\% hepatic system and 2\% renal system. ${ }^{21,22}$ Additional studies may be needed to investigate whether significant differences exist in the rate at which these tests are received by methotrexate users.

Measures assessing change in treatment, such as appropriate testing for new DMARD prescriptions (measures 0589 and 0590) or lab tests for a new RA patient altogether (measure 0601), were achieved by a greater proportion of patients (67.1\%-72.2\%) than the lone measure assessing annual lab testing by existing RA patients (measure 0592), which was only achieved by $56.0 \%-58.7 \%$. Further, measures 0589 and 0590, which examine baseline levels for serum creatinine and liver function testing, respectively, were met at a similar rate, which is in contrast to the differential rates of measure achievement in methotrexate users receiving these same tests (measures 0597 and 0599). It must be noted that the similar percentages observed for measures 0589 and 0590 are more in line with the percentage meeting measure 0599, indicating a reduction in liver function testing in patients receiving a new DMARD therapy compared with methotrexate users. The reason for this drop in liver function testing in patients receiving a new DMARD requires further study.

Measure 0592 assesses annual ESR and CRP testing and was achieved by the lowest proportion of patients (56.0\%-58.7\%). These tests determine the amount of inflammation present in the blood and are an indication of how well RA is being controlled. The low achievement rate of this measure, relative to 
other measures, may suggest that physicians are using other means to monitor disease activity, such as swollen and tender joint counts, patient reports, or composite disease activity measures. Conversely, these relatively lower rates of ESR and CRP testing may be indicative of a lack of consensus among physicians as to the best way to monitor disease activity. Elevations in ESR and CRP levels are an effective marker for inflammation, although their usefulness in tracking inflammation levels in individuals with chronic inflammation such as RA remains unclear. ${ }^{23}$ Discordant results have been reported among the 2 tests, and baseline levels can change with age. ${ }^{24-26}$ Further, the correlation of ESR and CRP levels with disease activity is not straightforward. ${ }^{27-34}$ As a result, there is a lack of consensus among physicians as to which test to use, as well as the overall accuracy of these tests as a marker of RA disease severity. $25-28,30,33,35,36$ Further study into physician practices and the clinical outcomes of RA disease activity monitoring will be able to clarify this issue. Regardless of the method used, increased monitoring of RA patients, even when the disease is well controlled, could allow for earlier identification of inflammatory flares and prompt treatment, hopefully preventing unnecessary tissue degradation and disease progression.

Although nearly $75 \%$ of this commercially insured sample of RA patients appear to be attaining these quality metrics on an annual basis, there remains a sizeable proportion of patients (approximately 25\%) who may be receiving suboptimal care. Based on the present study, it cannot be determined if this is largely patient- or physician-driven or some combination of both, but the undertreatment of RA has been shown to substantially increase health care costs..$^{37,38}$ Alternatively, it remains unclear if attainment of these measures translates into actual costs savings and improved clinical outcomes. A recent review found a small to moderate association between health care quality and cost, ${ }^{39}$ and there is some evidence that physicians affiliated with accountable care organizations can increase quality and reduce patient costs. ${ }^{40}$ Demonstrating a potential economic benefit of quality measure attainment may encourage health plans to mandate their adoption, which could improve patient care and reduce unnecessary health care spending. An additional examination of the relationship between health care expenditure and measure achievement is currently underway, although future studies should examine this relationship in other commercial health plans, in addition to the Medicare population, who are more likely to receive suboptimal care. ${ }^{11,12}$

\section{Limitations}

There were a number of limitations in the current study. The primary limitation was that the data source was administrative claims. Although administrative claims databases can provide access to extremely large sample sizes typically infeasible in clinical trials, and can also be adapted to answer a variety of complex research questions, ${ }^{41,42}$ coding inconsistencies across providers, general coding mistakes, and the dearth of clinical outcomes are some of their well-known inadequacies. ${ }^{43}$ Assessment of disease management through the use of patient medical data without direct patient or provider input can simplify analysis but is likely to result in an inherently less accurate conclusion. For instance, the disease activity measures with good or excellent reliability and validity as determined by the American College of Rheumatology include provider and patient assessments in the calculation. ${ }^{44}$ These measures were designed as tools for physicians to evaluate patients over time through analysis of administrative claims and medical records, making the trade-off of accuracy in disease management for ease of data analysis. This is not to state that these measures do not accurately reflect patient care; rather, they assess different aspects of care than measures that include physician or patient assessment. Although much of the information required to calculate these process measures may be gleaned from administrative claims, their aforementioned limitations may contribute to underperformance on the quality measures. Additionally, a number of measures assess laboratory testing, and whether the use of laboratory results or laboratory procedure codes are being used in the calculation of these measures, these tests may be ordered by physicians other than the treating rheumatologist, making it difficult to discern in a claims dataset whether patients are receiving these tests as part of their RA monitoring or for other comorbid conditions. One final limitation was the sample, which consisted of members of a single commercial health plan. It is unknown how results may generalize to members of other health plans or to the uninsured.

\section{Conclusions}

This is the first peer-reviewed examination of RA quality measure achievement assessed through administrative claims data of which the authors are aware. The present findings suggest that the majority of this commercially insured sample of RA patients is achieving these quality outcomes. Overall, measures assessing various aspects of existing DMARD usage, specifically methotrexate, were attained at high rates, while measures assessing new treatments, including new DMARD prescriptions or laboratory tests by new RA patients, were achieved by a slightly lower rate, albeit still the majority. Although results are encouraging, nearly $25 \%$ of patients may be receiving suboptimal care, suggesting a potential need for increased patient or physician awareness.

Additionally, it is presently unclear if increased measure attainment translates into genuine health care quality improvement and decreased health care costs. Further studies are needed to validate current findings, in addition to understanding whether attainment of these measures translates into better overall health or RA-related outcomes. Although these measures are designed to address common treatment pathways 
in RA, studies that demonstrate the value of these process measures to payers, accountable care organizations, and providers of RA treatment remain imperative. The first step in this process is to explore the relationship between process quality measure achievement and specific health care utilization and expenditure, which may ultimately determine metric relevance to health plan decision support.

\section{Authors}

JOSEPH TKACZ, MS, is Staff Scientist; BRENNA L. BRADY, PhD, is Project Director; and CHARLES RUETSCH, PhD, is President and CEO, Health Analytics, Columbia, Maryland. LORIE A. ELLIS, PhD, is Associate Director; ROXANNE MEYER, PharmD, is Manager; and SUSAN C. BOLGE, PhD, is Director, Health Economics \& Outcomes Research, Janssen Scientific Affairs, Horsham, Pennsylvania.

AUTHOR CORRESPONDENCE: Joseph Tkacz, MS, Health Analytics, 9200 Rumsey Rd., Ste. 215, Columbia, MD 21045. Tel.: 410.997.3314; Fax: 410.997.4545;

E-mail: joseph.tkacz@healthanalytic.com.

\section{DISCLOSURES}

Janssen Scientific Affairs funded this study. Ellis, Meyer, and Bolge are employees of Janssen Scientific Affairs and own stock in Johnson \& Johnson. Tkacz, Brady, and Ruetsch are employees of Health Analytics, which received research support from Janssen Scientific Affairs to conduct this study.

Study concept and design were contributed by Ellis, Meyer, Bolge, and Reutsch. Tkacz was primarily responsible for data interpretation, with assistance from the other authors. The manuscript was written and revised primarily by Tkacz and Brady, with assistance from the other authors.

\section{REFERENCES}

1. Aletaha D, Neogi T, Silman AJ, et al. 2010 Rheumatoid arthritis classification criteria: an American College of Rheumatology/European League Against Rheumatism collaborative initiative. Arthritis Rheum. 2010;62(9):2569-81.

2. Centers for Disease Control and Prevention. Prevalence of doctor-diagnosed arthritis and arthritis-attributable activity limitation-United States, 2007-2009. MMWR Morb Mortal Wkly Rep. 2010;59(39):1261-65.

3. Helmick CG, Felson DT, Lawrence RC, et al. Estimates of the prevalence of arthritis and other rheumatic conditions in the United States. Part I. Arthritis Rheum. 2008;58(1):15-25.

4. Centers for Disease Control and Prevention. National and state medical expenditures and lost earnings attributable to arthritis and other rheumatic conditions-United States, 2003. MMWR Morb Mortal Wkly Rep. 2007;56(1):4-7.

5. Kale MS, Bishop TF, Federman AD, Keyhani S. Trends in the overuse of ambulatory health care services in the United States. JAMA Intern Med. 2013;173(2):142-48.

6. National Research Council. (1990). Medicare: A Strategy for Quality Assurance, Volume 1. Washington, D.C.: The National Academies Press.

7. Scott DL. Biologics-based therapy for the treatment of rheumatoid arthritis. Clin Pharmacol Ther. 2012;91(1):30-43.
8. Bonafede MM, Fox KM, Johnson BH, Watson C, Gandra SR. Factors associated with the initiation of disease-modifying antirheumatic drugs in newly diagnosed rheumatoid arthritis: a retrospective claims database study. Clin Ther. 2012;34(2):457-67.

9. Lacaille D, Anis AH, Guh DP, Esdaile JM. Gaps in care for rheumatoid arthritis: a population study. Arthritis Rheum. 2005;53(2):241-48.

10. Westhoff G, Schneider M, Raspe H, et al. Advance and unmet need of health care for patients with rheumatoid arthritis in the German population-results from the German Rheumatoid Arthritis Population Survey (GRAPS). Rheumatology (Oxford). 2009;48(6):650-57.

11. Schmajuk G, Schneeweiss S, Katz JN, et al. Treatment of older adult patients diagnosed with rheumatoid arthritis: improved but not optimal. Arthritis Rheum. 2007;57(6):928-34.

12. Shipton D, Glazier RH, Guan J, Badley EM. Effects of use of specialty services on disease-modifying antirheumatic drug use in the treatment of rheumatoid arthritis in an insured elderly population. Med Care. 2004;42(9):907-13.

13. Chassin MR, Loeb JM. The ongoing quality improvement journey: next stop, high reliability. Health Aff (Millwood). 2011;30(4):559-68.

14. Adhikesavan LG, Newman ED, Diehl MP, Wood GC, Bili A. American College of Rheumatology quality indicators for rheumatoid arthritis: benchmarking, variability, and opportunities to improve quality of care using the electronic health record. Arthritis Rheum. 2008;59(12):1705-12.

15. National Committee for Quality Assurance, Physician Consortium for Performance Improvement, American College of Rheumatology. Rheumatoid arthritis: physician performance measurement set. July 2008. Available at: http://rheumatoidarthritis.semarthritisrheumatism.com/Content/PDFs/ RR-NCQA-Arthritis.pdf. Accessed December 14, 2014.

16. National Quality Forum. Measures, reports \& tools. Available at: http:// www.qualityforum.org/Measures_Reports_Tools.aspx. Accessed January 2, 2015

17. Charlson ME, Pompei P, Ales KL, MacKenzie CR. A new method of classifying prognostic comorbidity in longitudinal studies: development and validation. J Chronic Dis. 1987;40(5):373-83.

18. Singh JA, Furst DE, Bharat A, et al. 2012 update of the 2008 American College of Rheumatology recommendations for the use of disease-modifying antirheumatic drugs and biologic agents in the treatment of rheumatoid arthritis. Arthritis Care Res (Hoboken). 2012;64(5):625-39.

19. Zachariae H. Methotrexate side-effects. Br J Dermatol. 1990;122(Suppl 36): 127-33.

20. Jones KW, Patel SR. A family physician's guide to monitoring methotrexate. Am Fam Physician. 2000;62(7):1607-12, 1614.

21. Whiting-O'Keefe QE, Fye KH, Sack KD. Methotrexate and histologic hepatic abnormalities: a meta-analysis. Am J Med. 1991;90(6):711-16.

22. Widemann BC, Adamson PC. Understanding and managing methotrexate nephrotoxicity. Oncologist. 2006;11(6):694-703.

23. Watson J, Round A, Hamilton W. Raised inflammatory markers. BMJ. 2012;344:e454.

24. Costenbader KH, Chibnik LB, Schur PH. Discordance between erythrocyte sedimentation rate and $\mathrm{C}$-reactive protein measurements: clinical significance. Clin Exp Rheumatol. 2007;25(5):746-49.

25. Osei-Bimpong A, Meek JH, Lewis SM. ESR or CRP? A comparison of their clinical utility. Hematology. 2007;12(4):353-57.

26. Wolfe F. Comparative usefulness of C-reactive protein and erythrocyte sedimentation rate in patients with rheumatoid arthritis. J Rheumatol. 1997:24(8): 1477-85.

27. Blackburn WD Jr. Validity of acute phase proteins as markers of disease activity. J Rheumatol Suppl. 1994:42:9-13. 
28. Crowson CS, Rahman MU, Matteson EL. Which measure of inflammation to use? A comparison of erythrocyte sedimentation rate and C-reactive protein measurements from randomized clinical trials of golimumab in rheumatoid arthritis. J Rheumatol. 2009;36(8):1606-10.

29. Devlin J, Gough A, Huissoon A, et al. The acute phase and function in early rheumatoid arthritis. C-reactive protein levels correlate with functional outcome. J Rheumatol. 1997;24(1):9-13.

30. Grindulis KA, Calverley M, Constable TJ, Forster PJ, Ahmed ME, McConkey B. A comparison between clinical and laboratory tests in rheumatoid arthritis. Scand J Rheumatol. 1983;12(3):285-88.

31. Jansen LM, van der Horst-Bruinsma IE, van Schaardenburg D, Bezemer PD, Dijkmans BA. Predictors of radiographic joint damage in patients with early rheumatoid arthritis. Ann Rheum Dis. 2001;60(10):924-27.

32. Jansen LM, van Schaardenburg D, van Der Horst-Bruinsma IE, Bezemer PD, Dijkmans BA. Predictors of functional status in patients with early rheumatoid arthritis. Ann Rheum Dis. 2000;59(3):223-26.

33. Kay J, Morgacheva O, Messing SP, et al. Clinical disease activity and acute phase reactant levels are discordant among patients with active rheumatoid arthritis: acute phase reactant levels contribute separately to predicting outcome at one year. Arthritis Res Ther. 2014;16(1):R40.

34. Keenan RT, Swearingen CJ, Yazici Y. Erythrocyte sedimentation rate and C-reactive protein levels are poorly correlated with clinical measures of disease activity in rheumatoid arthritis, systemic lupus erythematosus and osteoarthritis patients. Clin Exp Rheumatol. 2008;26(5):814-19.

35. Mallya RK, de Beer FC, Berry H, Hamilton ED, Mace BE, Pepys MB. Correlation of clinical parameters of disease activity in rheumatoid arthritis with serum concentration of C-reactive protein and erythrocyte sedimentation rate. J Rheumatol. 1982;9(2):224-28.
36. Ward MM. Relative sensitivity to change of the erythrocyte sedimentation rate and serum C-reactive protein concentration in rheumatoid arthritis. J Rheumatol. 2004;31(5):884-95.

37. Kobelt G, Eberhardt K, Jonsson L, Jonsson B. Economic consequences of the progression of rheumatoid arthritis in Sweden. Arthritis Rheum. 1999;42(2):347-56.

38. Rat AC, Boissier MC. Rheumatoid arthritis: direct and indirect costs. Joint Bone Spine. 2004;71(6):518-24.

39. Hussey PS, Wertheimer S, Mehrotra A. The association between health care quality and cost: a systematic review. Ann Intern Med. 2013;158(1):27-34

40. Weeks WB, Gottlieb DJ, Nyweide DE, et al. Higher health care quality and bigger savings found at large multispecialty medical groups. Health Aff (Millwood). 2010;29(5):991-97.

41. Curtis JR, Chastek B, Becker L, et al. Further evaluation of a claimsbased algorithm to determine the effectiveness of biologics for rheumatoid arthritis using commercial claims data. Arthritis Res Ther. 2013;15(2):404.

42. Jean S, Candas B, Belzile É, et al. Algorithms can be used to identify fragility fracture cases in physician-claims databases. Osteoporos Int. 2012;23(2):483-501.

43. Tyree PT, Lind BK, Lafferty WE. Challenges of using medical insurance claims data for utilization analysis. Am J Med Qual. 2006;21(4):269-75.

44. Anderson J, Caplan L, Yazdany J, et al. Rheumatoid arthritis disease activity measures: American College of Rheumatology recommendations for use in clinical practice. Arthritis Care Res (Hoboken). 2012;64(5):640-47. 


\section{APPENDIX Drug Names and Procedural Codes Used}

\begin{tabular}{|c|c|c|}
\hline DMARDs & Brand Name & J Code \\
\hline Methotrexate & methotrexate, methotrexate Sodium, Trexall, Rheumatrex & J9250, J9260, J8610 \\
\hline Leflunomide & leflunomide, Arava & \\
\hline Azathioprine & Azathioprine, Imuran & $\mathrm{J} 7500, \mathrm{~J} 7501$ \\
\hline D-Penicillamine & Cuprimine, Depen & \\
\hline Intramuscular Gold & $\begin{array}{l}\text { gold thioglucose, gold sodium glucose, gold sodium thiomalate, auro- } \\
\text { thioglucose, Myochrysine, Auranofin, Ridaura, Solganol }\end{array}$ & \\
\hline Cyclosporine & cyclosporine, cyclosporine modified, Gengraf, Sandimmune, Neoral & $\mathrm{J} 7502, \mathrm{~J} 7515$ \\
\hline Cyclophosphamide & Cyclophosphamide & $\begin{array}{l}\text { J8530, J9070, J9080, J9090, J9091, J9092, } \\
\text { J9093, J9094, J9095, J9096, J9097 }\end{array}$ \\
\hline Sulfasalazine & sulfasalazine, Azulfidine & \\
\hline Hydroxychloroquine & $\begin{array}{l}\text { hydroxychloroquine, hydroxychloroquine sulfate, chloroquine phos- } \\
\text { phate, chloroquine HCL, Plaquenil }\end{array}$ & \\
\hline Minocycline & minocycline, minocycline hydrochloride, Minocin & \\
\hline Etanercept & Enbrel & \\
\hline Adalimumab & Humira & \\
\hline Anakinra & Kineret & \\
\hline Golimumab & Simponi & \\
\hline Certolizumab & Cimzia & \\
\hline Lab Test & CPT Code & \\
\hline Serum creatinine & $80047,80048,80050,80053,80069,82565,82575,84520,84525$ & \\
\hline Liver function & $80050,50053,80076,84450,84460$ & \\
\hline Erythrocyte sedimentation rate & 85651,85652 & \\
\hline C-reactive protein & 86140,86141 & \\
\hline Complete blood count & $\begin{array}{l}80050,80055,85007,85008,85025,85027,80047,80048,80050, \\
80053,80069,84520\end{array}$ & \\
\hline Blood urea nitrogen & 84525 & \\
\hline
\end{tabular}

